\title{
VANNOTE Vance, Women of White Earth (photographs and interviews by Vance Vannote with the editorial assistance from Janet Pratt), University of Minnesota Press, Minneapolis, 1999, 214 p.
}

\section{Emmanuel Désveaux}

\author{
(2) OpenEdition \\ Journals \\ Édition électronique \\ URL : http://journals.openedition.org/jsa/393 \\ DOI : $10.4000 /$ jsa. 393 \\ ISSN : $1957-7842$ \\ Éditeur \\ Société des américanistes \\ Édition imprimée \\ Date de publication : 1 janvier 2001 \\ Pagination : 407-409 \\ ISSN : 0037-9174 \\ Référence électronique \\ Emmanuel Désveaux, « VANNOTE Vance, Women of White Earth (photographs and interviews by Vance \\ Vannote with the editorial assistance from Janet Pratt), University of Minnesota Press, Minneapolis, \\ 1999, 214 p. », Journal de la Société des américanistes [En ligne], 87 | 2001, mis en ligne le 17 novembre \\ 2005, consulté le 22 septembre 2020. URL : http://journals.openedition.org/jsa/393 ; DOI : https:// \\ doi.org/10.4000/jsa.393
}

Ce document a été généré automatiquement le 22 septembre 2020.

(C) Société des Américanistes 
VANNOTE Vance, Women of White Earth (photographs and interviews by Vance Vannote with the editorial assistance from Janet Pratt), University of Minnesota Press, Minneapolis, 1999, $214 \mathrm{p}$.

\author{
Emmanuel Désveaux
}

1 Voici un ouvrage qui a l'allure d'une galerie de portraits. L'auteur se propose en effet de présenter quarante trois personnalités féminines issues de la réserve de White Earth et qui parfois y habitent encore. Chacune d'entre elles a droit à un traitement similaire, en forme de diptyque associant l'image et le texte. À une photographie en noir et blanc, rayonnante de présence humaine - Vance Vannote se veut un lointain disciple de Daguerre et, plus sûrement encore, de Curtis - répondent ainsi une brève note biographique et un texte de trois à quatre pages dans lequel la personne (re)présentée se raconte elle-même sur un mode à mi-chemin entre l'autobiographie et la confession. Ce dernier texte résulte d'un entretien avec l'auteur.

2 Sur la carte, White Earth apparaît comme la plus grande réserve indienne du Minnesota. C'est un domaine enchanté de forêts généreuses et de lacs, certains majestueux, certains secrets. Malheureusement, c'est une réserve presque virtuelle, en ce sens que les Ojibwa qui la possèdent en ont pratiquement perdu tout le foncier au fil des décennies. Poussés par le besoin, ils ont été progressivement conduits à vendre la terre, d'abord à des compagnies d'exploitation forestière, puis à des citadins venus de Minneapolis ou de Fargo y bâtir des résidences secondaires. De sorte que seules quelques agglomérations, telles White Earth, Mahnomen ou Bemidji, donnent à entrevoir une présence indienne singulièrement évanescente par ailleurs : plus grande pauvreté de l'habitat, moindre blondeur de la couleur des cheveux des habitants - ce 
sont pour l'essentiel des paysans scandinaves qui ont colonisé au XIXe siècle le nord-est du Minnesota.

3 Écoutons maintenant (lisons plutôt) le témoignage de Berverly Warren :

Enfant, ma grand-mère avait été emmenée de chez elle pour être envoyée dans un pensionnat. Quand je l'ai connue, elle se teignait toujours les cheveux en blond platine. J'ignorais totalement qu'elle parlait indien jusqu'à cet été de mes vingtdeux ans que j'ai entièrement passé chez elle. Un jour, elle commença à entonner des chants indiens. Je lui ai dit : « Grand-Mère, tu connais des chants indiens ? " Elle répond: "Avant, ce n'étaient pas vraiment des choses à savoir». Je dis alors: «Mais, Grand-Mère, j'ignorais que tu parlais indien. Pourquoi ne m'as-tu pas appris?». Elle dit: "Ma fille, je pensais que c'était mieux pour moi de ne pas le faire ".

4 Au fond, l'histoire de White Earth en tant que communauté amérindienne est d'une extraordinaire banalité : c'est celle d'une dépossession, dépossession matérielle d'abord que symbolise jusqu'à la caricature cette situation d'une réserve dont les "bénéficiaires" ne bénéficient plus de rien, dépossession immatérielle ensuite, puisque, un siècle durant, toutes les formes de «développement " économique, d'une part, toutes les institutions, d'autre part, ont concouru afin de décourager les gens de parler et, plus grave encore, de transmettre leur langue. En bref, tout a été fait pour les priver de leur culture initiale, et ce, avec l'incontestable succès que l'on sait.

5 Comme l'on peut s'y attendre, l'amertume hante ce livre, suinte de chacune de ses pages. Toutefois, grâce au talent de Vannote et de Janet Pratt - dans sa façon à lui de conduire les entretiens ou dans leur travail commun de réécriture - Women of White Earth parvient à la contenir, à éviter que le ressentiment ne devienne une déferlante fixe, comme c'est trop souvent le cas dans des ouvrages de ce type. Du coup, un autre message passe. La somme de ces textes et de ces images de femmes qui appartiennent à toutes les classes d'âge, de l'adolescente à l'« aînée ", nous raconte alors une histoire qui n'est pas seulement celle de la perte originale, de la blessure toujours à vif, mais aussi celle d'une relative guérison, d'une réconciliation possible avec le monde. La réussite de la majorité d'entre elles, dans des secteurs professionnels extrêmement divers, est systématiquement mise en avant. De très nombreux métiers figurent ici - de l'assistante sociale à la gardienne de parc naturel en passant par celui de laborantine, d'infirmière, de femme d'affaire, d'artiste, d'avocat, d'employée de casino, de militante (si tant est que dans ce dernier cas, on puisse parler de "métier »)... À cet égard, nul doute que ce livre participe d'une idéologie profondément américaine, laquelle nous apparaît bien entendu d'une grande naïveté. L'éventail des possibles offert à chacun est très large ; tout est possible dans la société nord-américaine dès lors que l'individu se décide à prendre en main son destin et sait saisir les occasions qui se présentent à lui. $\mathrm{Au}$ fond, cette double orientation du texte, individuelle - on braque le projecteur tour à tour sur des individus - et quasi thérapeutique - montrer comment à travers leur parcours professionnel, ces femmes ont accédé à une certaine sérénité -, ne doit pas nous surprendre dans la mesure où Vannote est psychologue. On conçoit bien alors la qualité de la confiance qu'il a réussi à instaurer entre lui et ses interlocutrices.

On pourra en revanche s'étonner du fait qu'il soit un homme. On s'attendrait plutôt à une femme dans cette position de Grand écoutant, compte tenu du parti pris de l'ouvrage de ne s'intéresser qu'aux femmes de White Earth. À première vue en effet, on se situe dans le courant typiquement américain des women studies. De fait, la perplexité du lecteur s'accroît - mais peut-être qu'au contraire tout cela est d'une logique 
parfaite - lorsque il s'aperçoit que toutes ces femmes ne parlent pas - ou très peu - des hommes. Toutes ces femmes restent étrangement muettes sur les hommes qui ont nécessairement croisé leur existence, du côté de l'affinité, maris, compagnons, pères de leurs enfants, mais aussi du côté de la consanguinité, puisque non seulement la figure $\mathrm{du}$ frère est absente, mais, fait plus troublant, également celle du fils. Ces femmes, en effet, ont soit uniquement des " enfants ", soit elles mentionnent explicitement leur(s) fille(s). Seule émerge occasionnellement de ce non-dit l'évocation d'une figure paternelle - ou, de préférence d'ailleurs, grand-paternelle. Joan Staples, une des femmes parmi les plus lucides portraiturées dans ce recueil, nous livre la clé de cette étrange oblitération du masculin en soulignant l'extrême fragilité des hommes dans l'univers contemporain, en comparaison avec les femmes (p. 150).

7 Dans la société traditionnelle, ils étaient voués en priorité à la production de la mort, tantôt comme chasseurs, tantôt comme guerriers. Aujourd'hui, aucun rôle ne leur est dévolu. Aux femmes, il reste précisément la maternité, la responsabilité de perpétuer le groupe, quand bien même celui-ci se délite au fur et à mesure que sa culture distinctive s'érode. Cela affleure à plusieurs reprises à travers les entretiens : l'indianité dont il est ici question ne tient parfois qu'à très peu de chose, ne tient qu'à un fil, celui-ci n'étant autre que, sans jeu de mot, le cordon ombilical. Cette indianité découle parfois en effet simplement du fait d'être enregistré sur les fichiers d'état civil de la tribu. Anciennement, chez les Ojibwa, c'était au père qu'il revenait de recueillir le cordon ombilical de son enfant et d'aller le disposer en forêt afin qu'il soit mangé par une bête sauvage, idéalement peut-être celle qui servait d'emblème tutélaire à son propre clan. Par ce geste, il affirmait l'appartenance de son enfant à ce clan. Il semble que cet usage soit bel et bien oublié. D'ailleurs, personne ne se soucie plus, à travers tout ce livre, d'évoquer l'ancien système clanique patrilinéaire qui régissait la société ojibwa... Tout se passe comme si aujourd'hui la réalité sociale de White Earth se limitait à ses femmes; les hommes, dépouillés de toutes leurs prérogatives, ne sont plus que des ombres à leur côté, victimes toutes désignées de l'alcoolisme, autre thème qui parcourt in petto Women of White Earth. Par là même, cet ouvrage est à sa façon, ô combien poignante, aussi un grand livre d'ethnographie, et ce, en dépit de ses intentions évidentes de ne pas en être un.

\section{AUTEURS}

\section{EMMANUEL DÉSVEAUX}

EHESS, Paris 